\title{
Influence of coil characteristics on sensor sensitivity of permeability testing technology based on constant current source
}

\author{
Ren Xianzhi', a, Ren Shangkun ${ }^{2, b}$ \\ ${ }^{1}$ Key Laboratory of Nondestructive Testing of Ministry of Education, Nanchang \\ Hangkong University, Nanchang 330063, Jiangxi, China \\ ${ }^{2}$ Key Laboratory of Nondestructive Testing of Ministry of Education, Nanchang \\ Hangkong University, Nanchang 330063, Jiangxi, China \\ a2089564480@qq.com, brenshangkun@yeah.net
}

Keywords: nondestructive testing; permeability testing; excitation coil; detection coil; detection sensitivity

Abstract. The permeability testing technology is a kind of non-destructive testing method, which based on the change of permeability, can detect the stress concentration state and the degree of fatigue damage for ferromagnetic samples. The detection sensor based on the constant current source has higher detection sensitivity, which overcomes the current negative feedback effect caused by the change of the magnetic circuit impedance of the ordinary constant voltage source. According to the principle of permeability detection, the influence of the number of turns of excitation coil, the number of turns of detection coil on the detection sensitivity were studied. The study shows that the detection sensitivity increases first with the increase of the number of turns of the excitation coil and then approaches the constant. The detection sensitivity increases as the number of turns of the detection coil increase, which is approximately linear relationship. The research can provide reference for the development of high sensitivity magnetic permeability detector.

\section{Introduction}

Permeability testing technology is a new detection method, which can detect the stress concentration and fatigue damage of the whole or regional ferromagnetic components early and effectively evaluate its residual life[1]. Among them, the detection sensor is an important part of the detection system, its performance determines the sensitivity and detection quality of magnetic permeability detection method[2]. Therefore, the design of sensor based on magnetic permeability detection technology has an important practical significance[3]. For permeability testing technology, there are many factors that affect the performance of the sensor, For example, core selection, coil winding method and enameled wire selection[4].

At present, the evaluation methods of the micro-defects such as fatigue damage, stress concentration and heat treatment quality of the ferromagnetic specimen include metal magnetic memory test[5,6], Barkhausen effect test[7], acoustic emission test[8], ultrasonic stress test[9], the radiation stress detection method[10] and so on, but there are limitations in terms of detection reliability, detection resolution and practical effect, and need to be further studied. This paper attempts to establish a model of the number of turns of excitation coil, the number of turns of detection coil and detection sensitivity using magnetic permeability testing technology[11,12], optimize the detection sensor system from the detection signal and excitation signal, and will be of great significance to the inspection of micro-defects such as stress concentration, fatigue damage or heat treatment of the ferromagnetic components in the project.

\section{Analysis of the relationship between the detection signal and permeability}

The theoretical basis of the permeability testing technology is the microscopic force effect and electromagnetic induction principle. When the detection probe which is fed into the alternating signal 
approch the seized ferromagnetic workpiece, the probe and the seized ferromagnetic workpiece form a closed magnetic circuit. When there is stress concentration and fatigue damage inside the ferromagnetic specimen, according to the excitation effect, the magnetic flux density changes when the magnetic flux in the closed magnetic circuit passes through the part. According to the principle of electromagnetic induction, the output signal of the detection coil changes with the change of the magnetic flux density inside the magnetic core. By extracting the value of the induced voltage output by the detection coil, can reflect the change of the initial permeability of the ferromagnetic material. Test probe structure shown in Figure 1.

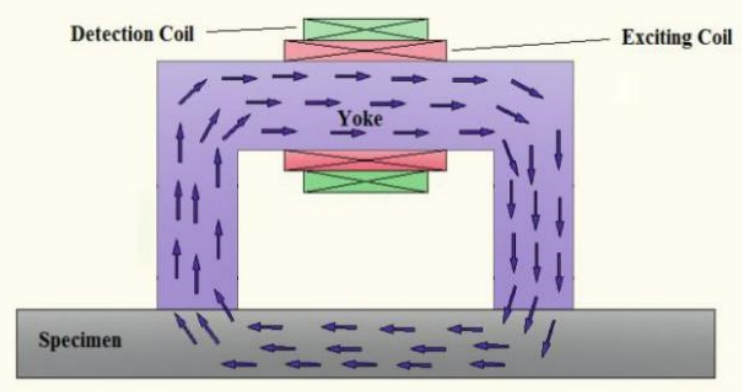

Fig. 1 Sensor structure diagram

Select the constant current source as the excitation source. Based on magnetic circuit loop theorem and magnetic Ohm's Law: $i=\phi R_{m}, i$ express excitation current of the yoke, $\phi$ express the magnetic flux generated by the single-turn excitation current, $R_{m}$ express the magnetic resistance of full magnetic circuit. Therefore, $\phi=i / R_{m}, R_{m}$ consist of $R_{m o u t}$ and $R_{\min }$, which expressed the magnetic resistance of air part and magnetic resistance of specimens respectively in the magnetic circuit. Therefore, $\phi=i / R_{m}=i /\left(R_{\text {mout }}+R_{\min }\right) \quad, \phi==i /\left[R_{\text {mout }}+L /(\mu S)\right], \mu$ express permeability of specimen, $\mathrm{L}$ is the length of specimen, $\mathrm{S}$ express the average equivalent cross-sectional area. According to Maxwell second equation:

$$
\begin{aligned}
& \int E \cdot d l=-\int_{s} \frac{\partial B}{\partial t} \cdot d s \text {, flux density } B=\phi / S \\
& \text { output signal can be obtained: } u_{\text {out }}=N_{2} \oint E \cdot d l=-\frac{N_{2}}{S} \int \frac{\partial \phi}{\partial t} \cdot d s \\
& \qquad u_{\text {out }}=-\frac{N_{2}}{S} \int \frac{\partial \phi}{\partial t} \cdot d s=-N_{2} \frac{1}{R_{m_{\text {out }}}+L /(\mu S)} \frac{d i}{d t}
\end{aligned}
$$

In equation (2), $\mathrm{N}_{1}$ and $\mathrm{N}_{2}$ express the number of turns of the excitation coil and the detection coil, respectively. Formula (2) indicates that when the permeability $\mu$ of the specimen to be changed, the output signal can change correspondingly. Negative express the phase relation of current and voltage. It can be seen that the detection voltage signal is closely related to the permeability $\mu$ of the test piece and can directly reflect the differential permeability of the test piece. By detecting the induced signal, the change of permeability of the specimen can be determined, and then reflect the internal structure state of the ferromagnetic components.

\section{Influence of coil characteristics on detection sensitivity}

\section{The relationship model of excitation coil turns $(\mathrm{N})$ and detection sensitivity}

Maintain the number of turns of the detection coil is 300 , the excitation current $I=0.06 \mathrm{App}$, the excitation frequency $\mathrm{f}=1000 \mathrm{~Hz}$, the relationship between the detection sensitivity and the number of turns of the excitation coil was experimentally studied, as shown in Figure 2 (a). Among them, the measured voltage signal is peak-to-peak (Vpp). As can be seen from Fig. 2 (a), within a certain 
number of turns of the excitation coil, the detection sensitivity increases with the number of turns of the excitation coil, showing that the growth rate is large first, then small, and tends to be constant in the last. The influence of the number of turns of the excitation coil on the detection signal includes two factors. One is the increase of the number of turns of the excitation coil, and the increase of the magnetic field strength linearly, resulting in an increase of the detection signal. Second, as the number of turns of the excitation coil increases, the magnetic flux in the yoke gradually becomes saturated and tends to be constant. When the number of turns of the excitation coil $\mathrm{N}=400$, the detection sensitivity tends to be the highest, set $\mathrm{N}=400$ for the optimum excitation coil turns.

Figure 2 (b) shows the relationship of the detection sensitivity with frequency changes under the different excitation coil turns. As can be seen from Figure 2 (b), as the number of turns of the excitation coil increases, the optimum frequency shifts to low frequencies, the first extreme point detection signal gradually increases and the second extreme point the detection signal is substantially unchanged.

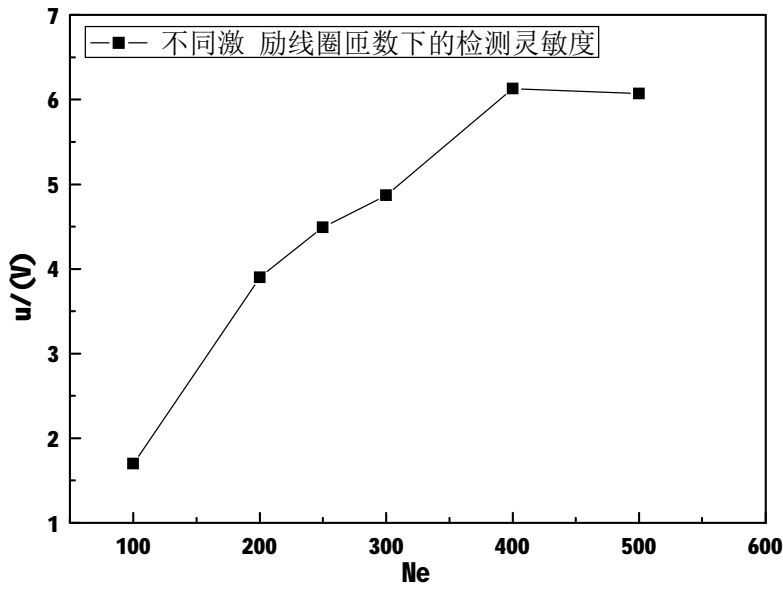

(a)

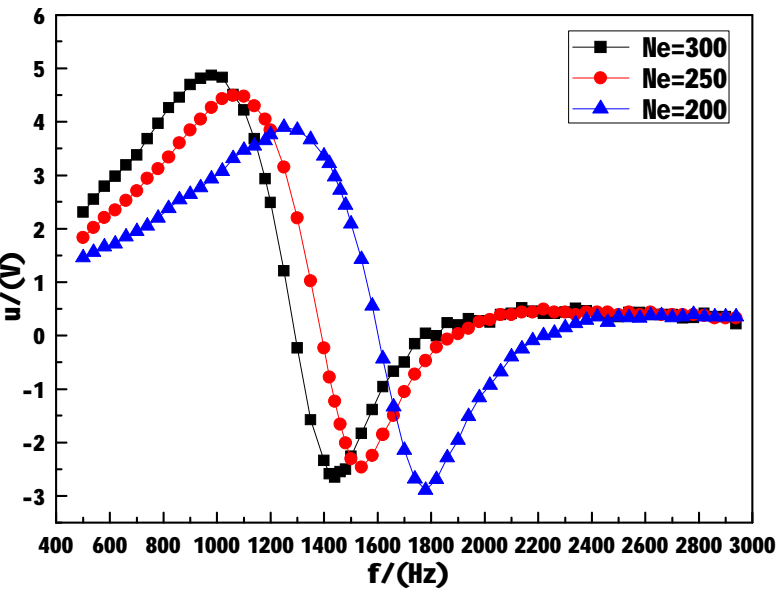

(b)

Fig. 2 (a) The curve of detection sensitivity changing with turns of excitation coil

(b) The curve of detection sensitivity changing with the frequency under different turns of excitation coil

\section{The relationship model of detection coil turns $(\mathrm{N})$ and detection sensitivity}

Maintain the number of turns of the excitation coil is 300 , the excitation current $\mathrm{I}=0.06 \mathrm{App}$, the excitation frequency $\mathrm{f}=1000 \mathrm{~Hz}$, the relationship between the detection sensitivity and the number of turns of the detection coil was experimentally studied, as shown in Figure 3 (a). As can be seen from Fig. 3 (a), within a certain number of turns of the detection coil, and the detection sensitivity shows a linear upward trend as the number of turns of the detection coil increase. Figure 3 (b) shows the relationship of the detection sensitivity with frequency changes under different turns of the detection coil. As can be seen from Fig. 3 (b), the optimal frequency has nothing to do with the number of turns of the detection coil, does not vary with the number of turns of the detection coil. 


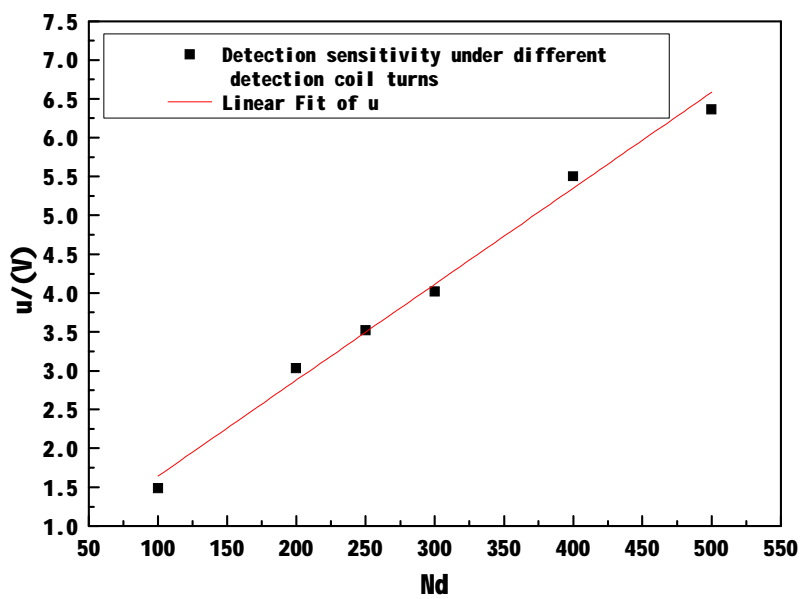

(a)

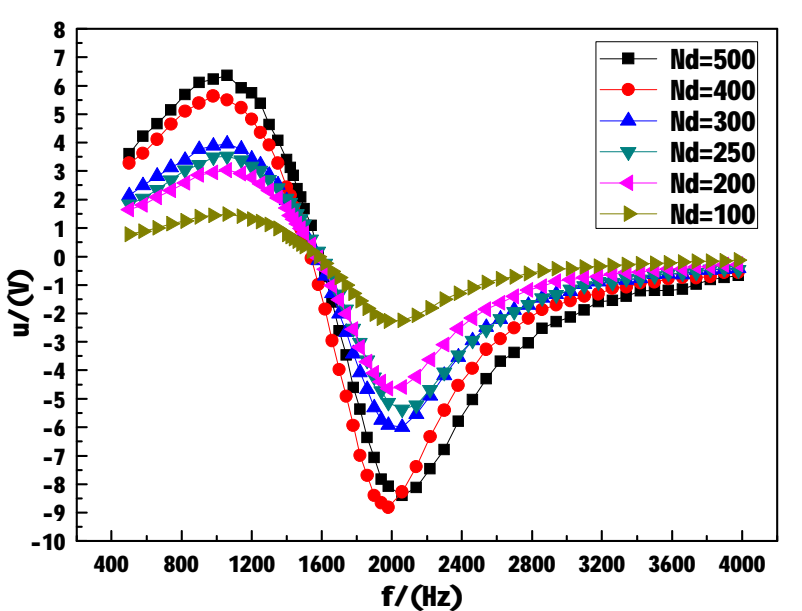

(b)

Fig. 3 (a) The curve of detection sensitivity changing with turns of detection coil

(b) The curve of detection sensitivity changing with the frequency under different turns of detection coil

\section{Conclusions}

Through the design of sensor of permeability testing technology, the influence of the number of turns of excitation coil, the number of turns of the detection coil on the detection sensitivity were studied, the following conclusions can be obtained:

(1) In a certain number of turns of excitation coil, the optimal excitation frequency, detection sensitivity is related to the number of turns of the excitation coil turns . The detection sensitivity increases with the increase of the number of excitation coil turns, then the saturation tends to a constant value. As the number of excitation coil turns increases, the optimum frequency shifts to low frequencies.

(2) Detection sensitivity is related to the number of turns of the detection coil, and increases linearly with the increase of the number of turns of the detection coil. The optimum detection frequency is independent of the number of turns of the detection coil and does not change with the number of turns of the detection coil.

\section{References}

1] Ren Shangkun, Ou Yangchun, Fu Renzhen, Fu Yuewen. Studies on stress-magnetism coupling effect for 35 steel components[J]. Insight: Non-Destructive Testing and Condition Monitoring, 2010, 52(6):305-309.

[2] Ren S K, Fu R Z, Li X L, et al. Influences of environmental magnetic field on stress-magnetism effect for 20 steel ferromagnetic specimen[J]. Insight: Non-Destructive Testing and Condition Monitoring, 2009, 51(12):672-675.

[3] Rabung M, Altpeter I, Boller C, et al. Non-destructive evaluation of the micro residual stresses of IIIrd order by using micro magnetic methods[J]. Ndt \& E International, 2014, 63(4):7-10.

[4] Zeng J, Su L, Xu L, et al. Research on the stress measurement of steel plate based on inverse magnetostrictive effect[J]. Journal of Mechanical Engineering, 2014, 50(8):17-22.

[5] Dong L, Xu B, Dong S, et al. Variation of stress-induced magnetic signals during tensile testing of ferromagnetic steels[J]. Ndt \& E International, 2008, 41(3):184-189.

[6] Wang Z D, Yao K, Deng B, et al. Theoretical studies of metal magnetic memory technique on magnetic flux leakage signals[J]. Ndt \& E International, 2010, 43(4):354-359. 
[7] Franco F A, Padovese L R. NDT flaw mapping of steel surfaces by continuous magnetic Barkhausen noise: Volumetric flaw detection case[J]. Ndt \& E International, 2009, 42(8):721-728.

[8] R'Mili M, Moevus M, Godin N. Statistical fracture of E-glass fibres using a bundle tensile test and acoustic emission monitoring $[\mathrm{J}]$. Composites Science \& Technology, 2009, 68(7):1800-1808.

[9] Bossi R H, Giurgiutiu V. Nondestructive testing of damage in aerospace composites[M]// Polymer Composites in the Aerospace Industry. 2015:413-448.

[10] Gao W, Hu Y, Mu X, et al. Real-time detection and segmentation of submerged-arc welding defects in X-ray radiography images[J]. Yi Qi Yi Biao Xue Bao/chinese Journal of Scientific Instrument, 2011, 32(6):1215-1224.

[11] Vertesy G, Uchimoto T, Tomáš I, et al. Nondestructive characterization of ductile cast iron by magnetic adaptive testing[J]. Journal of Magnetism \& Magnetic Materials, 2010, 322(20):3117-3121.

[12] Tomáš I. Non-destructive magnetic adaptive testing of ferromagnetic materials[J]. Journal of Magnetism \& Magnetic Materials, 2004, 268(1):178-185. 